\title{
ПОСТРОЕНИЕ ШКАЛ ЭКВИВАЛЕНТНОСТИ ДЛЯ ОТДЕЛЬНЫХ ТЕРРИТОРИАЛЬНЫХ ЕДИНИЦ АРМЕНИИ
}

\author{
Евгения Базинян, \\ Ассистент кафедры «Экономико-математических методов» АГЭУ, \\ кандидат экономических наук
}

DOI: https://doi.org/10.31435/rsglobal_ijite/31012019/6333

\begin{tabular}{l} 
ARTICLE INFO \\
Received 24 November 2018 \\
Accepted 26 January 2019 \\
Published 31 January 2019 \\
\hline
\end{tabular}

\section{KEYWORDS}

poverty level, poverty indicators, population differentiation indicators based on income and expenses, equivalence scale. \begin{abstract}
An important indicator of the welfare and living standards of the population is the level of the poor population in the given country. As practice shows, in economically developed countries the policy in the population income sphere is one of the key elements of economic management. In countries with transforming economies such as Armenia, the problem of population income regulation is extremely topical. In resolving many of social policy issues the need to compare the living standards of families with different structures arises. The work is devoted to the construction of equivalence scales which take into account the actual purchasing power differences of similar incomes in different size households.
\end{abstract}

Citation: Yevgenya Bazinyan. (2019) The construction of equivalence scales for separate territorial units in Armenia. International Journal of Innovative Technologies in Economy. 1(21). doi: 10.31435/rsglobal_ijite/31012019/6333

Copyright: (C) 2019 Yevgenya Bazinyan. This is an open-access article distributed under the terms of the Creative Commons Attribution License (CC BY). The use, distribution or reproduction in other forums is permitted, provided the original author(s) or licensor are credited and that the original publication in this journal is cited, in accordance with accepted academic practice. No use, distribution or reproduction is permitted which does not comply with these terms.

На современном этапе развития экономики Армении проблемы повышения уровня жизни населения и исследования факторов, определяющие его динамику, очень важны. От их решения во многом зависит направленность и темпы дальнейших преобразований в стране и, в конечном счете, экономическая и политическая стабильность в обществе. Решение этих проблем требует определенной политики, выработанной государством, центральным моментом которой был бы человек, его благосостояние, физическое и социальное здоровье. Именно поэтому все преобразования, которые так или иначе могут повлечь изменение уровня жизни, вызывают большой интерес у самых разнообразных слоев населения.

Различные измерители уровня бедности и показатели дифференциации населения по доходам и расходам относятся к ключевым индикаторам качества проводимой социальной политики и используются, в частности, при построении адресной социальной помощи малоимущим слоям населения, нацеленной на максимальное снижение социальной напряженности в обществе.

При сравнении уровня жизни домохозяйств нельзя не принимать во внимание проблемы, связанные с сопоставлением расходов и доходов семей различного размера, социально-демографического состава и территориальной принадлежности. Эта проблема вызывает необходимость построения шкал эквивалентности, позволяющих корректно учитывать различия в реальной покупательной силе одинаковых доходов в домохозяйствах различных размеров за счет экономии, полученной от ведения большого хозяйства. Обоснованно судить об уровне жизни населения можно лишь владея данными о количестве и соотношении групп домохозяйств, обладающих различным уровнем жизни. И. Дмитричев в 
работе «Статистика уровня жизни населения ${ }^{1}$ пишет, что основной информационной базой для исследования уровня жизни населения является статистика домашних хозяйств (ДХ).

Различия между ДХ в размере и по составу вызывают необходимость специальных расчетов, позволяющих оценить благосостояние ДХ с помощью так называемых «шкал эквивалентности. Эти шкалы дают возможность оценивать доходы ДХ в расчете на условного члена. Этот подход помогает корректно учесть различия в реальной покупательной силе доходов за счет экономии от ведения большого хозяйства ${ }^{2}$.

На практике при построении шкал эквивалентности чаще всего берется за основу анализ потребительского поведения. В странах с большим уровнем бедности в роли источника экономии предполагаются расходы на питание, нежели расходы на общесемейные нужды. Причина в том, что в этих странах расходы на питание составляют большую долю в прожиточном минимуме, его непродуктовая часть настолько мала, что практически не предполагает приобретения предметов длительного пользования. В этих условиях источником экономии обычно служит замена относительно дорогих полуфабрикатов дешевыми продуктами питания, прошедшими минимальную предварительную обработку. Еще один источник экономии состоит в том, что закупка продуктов питания, особенно скоропортящихся, в больших количествах обходится дешевле.

Воспользовавшись данными бюджетного обследования ДХ Армении 2016 3 года и применяя множественный регрессионный анализ в модели спроса ${ }^{4}$ исследовалась зависимость среднедушевых общих расходов $(y)$ от величины среднедушевых доходов $(x)$ и численности ДХ $\left(N_{l}, l=2,3,4,5,6\right)^{5}$.

Для разработки шкалы эквивалентности в данной статье используется регрессионный анализ, с помощью которого выявляется зависимость среднедушевых общих расходов от среднедушевых доходов и численности домохозяйства, по отдельным территориальным единицам:

$$
\ln Y_{i}^{j}=c_{0}+c_{1} \ln X_{i}^{j}+c_{2} N 2_{i}+c_{3} N 3_{i}+\ldots+c_{6} N 6_{i}+\varepsilon_{i},
$$

где $\ln Y_{i}^{j}$ - логарифм душевого расхода $i$-го домохозяйства $j$-й территориальной единицы $\left(j=1,2,3: j=1\right.$ - Ереван, $j=2$ - другие города и $j=3$-сельские местности); $\ln X_{i}^{j}-$ логарифм душевого дохода $i$-го домохозяйства $j$-й территориальной единицы; $N 2_{i}, N 3_{i}, \ldots, N 6_{i}-$ дихотомические переменные, отображающие влияние размера домашнего хозяйства (ДХ) на среднедушевой расход. Они имеют следующую структуру:

$$
\begin{gathered}
N 2_{i}=\left\{\begin{array}{c}
1, \text { если } i-\text { ое домохозяйство состоит из двух человек; } \\
0, \text { в противном случае, } \\
\vdots
\end{array}\right. \\
N 6_{i}=\left\{\begin{array}{c}
1, \text { если } i-\text { ое домохозяйство состоит из шести и более человек; } \\
0, \text { в противном случае. }
\end{array}\right.
\end{gathered}
$$

После исследования регрессионных моделей для трех территориальных единиц получены следующие оценки логарифмов душевых расходов:

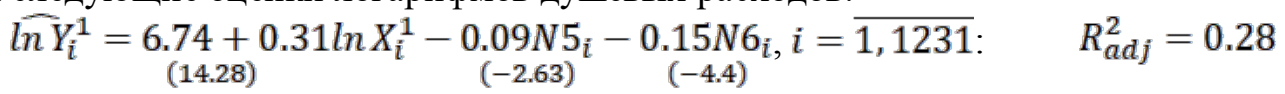

$$
\begin{aligned}
& \widehat{\ln Y_{i}^{2}}=\underset{(16.72)}{5.95+35 \ln X_{i}^{2}}-\underset{(-2.74)}{0.10 N 4_{i}}-\underset{(-4.67)}{0.19 N 5_{i}}-\underset{(-6.02)}{0.25 N 6_{i}}, i=\overline{1,1231}: R_{\text {adj }}^{2}=0.33 \\
& \widehat{\ln Y_{i}^{3}}=\underset{(18.56)}{6.04}+0.36 \ln X_{i}^{3}-\underset{(-3.27)}{0.24 N 2_{i}}-\underset{(-4.69)}{0.36 N 3_{i}}-\underset{(-7.27)}{0.51 N 4_{i}}-\underset{(-9.51)}{0.68 N 5_{i}}-\underset{(-12.57)}{0.89 N 6_{i}}, \\
& i=\overline{1,1637}: R_{\text {adj }}^{2}=0.41
\end{aligned}
$$

\footnotetext{
${ }^{1}$ Дмитричев И. И, "Статистика уровня жизни населения", Москва, Госкомстат РФ, 1995

${ }^{2}$ Jesus Fernandez-Villaverde, Dirk Krueger, "Consumption over the Life Cycle: Some Facts from Consumer Expenditure Survey Data", 2002, p. 60

${ }^{3}$ www.armstat.am

${ }^{4}$ Джеффри Д. Сакс, Ларрен Б. Макроэкономика в глобальном экономике. - Ереван, 2002 г.

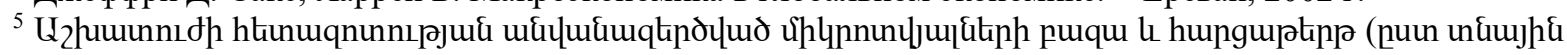

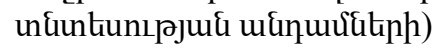


Скорректированные коэффициенты детерминации получились невысокими, что объясняется не включением в модели других переменных, связанных с душевыми расходами. Однако, исходя из содержания поставленной задачи и того факта, что все коэффициенты при зависимых переменных статистически значимы, считаем возможным использовать их для построения шкалы эквивалентности (в скобках приведены $t$-значения соответствующих коэффициентов.).

Исходя из содержательной интерпретации решаемой задачи и того факта, что все коэффициенты объясняющих переменных статистически значимы (гипотезы о равенстве нулю коэффициентов отвергаются с 0.05 уровнем значимости), эти коэффициенты были использованы для построения шкалы эквивалентности.

Из уравнений (1), (2) и (3) видно, что все коэффициенты при переменных $N 2, N 3, \ldots, N 6$ отрицательны. Это означает, что при фиксированном значении душевого дохода, душевые расходы в домохозяйствах большего размера уменьшаются, что подтверждает факт наличия экономии на больших домохозяйствах. Однако, в (1) и (2) эффект экономии проявляется начиная не от $N 2$, как в (3), а соответственно от $N 5$ и $N 4$. Остальные дихотомические переменные были статистически не значимыми в (1) и (2) и поэтому были исключены из них.

Таким образом, результаты регрессионного анализа подтверждают наличие экономии в больших домохозяйствах, однако если в столице и в других городах такая экономия проявляется только в домохозяйствах с четырьмя и более членами, то в сельских местностях она дает о себе знать уже в домохозяйствах с двумя и более членами.

На основе формул (1), (2) и (3) была построена шкала эквивалентности:

Таблица 1. Шкала эквивалентности

\begin{tabular}{|l|c|c|c|c|c|c|}
\hline \multirow{2}{*}{} & \multicolumn{7}{|c|}{ Размер домашних хозяйств } \\
\cline { 2 - 7 } & $\mathbf{1}$ & $\mathbf{2}$ & $\mathbf{3}$ & $\mathbf{4}$ & $\mathbf{5}$ & 6 и более \\
\hline В Ереване & 1 & 2 & 3 & 4 & 4,91 & 5,77 \\
\hline В других городах & 1 & 2 & 3 & 3,91 & 4,82 & 5,76 \\
\hline В сельских местностях & 1 & 1,79 & 2,49 & 3,09 & 3,6 & 4,01 \\
\hline
\end{tabular}

Коэффициенты означают, что, например, если домохозяйство в сельской местности с одним членом получает одну единицу дохода и при этом имеет конкретный уровень потребления, то домохозяйству с двумя членами, для достижения того же уровня потребления достаточно иметь 1.79 единиц дохода вместо 2, как в случае домохозяйств столицы или других городов и т.д.

Таким образом, для сопоставления реального уровня жизни домохозяйств различного размера в отдельных территориальных единицах предлагаем привести сопоставляющие характеристики к эквивалентному уровню с помощью полученной шкалы эквивалентности.

\section{ЛИТЕРАТУРА}

1. Кукушкин Д. К., Подузов А. А., Шкала эквивалентности как инструмент измерения уровня жизни // Проблемы прогнозирования, 2000, № 4, с. 42-59.

2. Овчарова Л. Н. Теоретико-методелеогические вопросы определения и измерения бедности // В центре внимания// SPERO | №16 Весна-Лето 2012.

3. Подузов А. А. Измерение бедности. Зарубежный опыт // Проблемы прогнозирования, 1996, № 5.

4. Румянцева Е. Е. Стратегия преодоления бедности: монография, Москва, Берлин: Директ-Медиа, 2015, 444 c.

5. Income distribution in OECD countries. Evidence from Luxemburg income study, OECD, Paris, 1995

6. www.armstat.am 